\title{
Document Ranking using Customizes Vector Method
}

\author{
Priyanka Mesariya \\ Computer Engineering, Gujarat Technological \\ University, India
}

\author{
Nidhi Madia \\ Computer Engineering, Gujarat Technological \\ University, India
}

\section{ABSTRACT}

Information retrieval (IR) system is about positioning reports utilizing client's question and get the important records from extensive dataset. Archive positioning is fundamentally looking the pertinent record as per their rank. Document ranking is basically search the relevant document according to their rank. Vector space model is traditional and widely applied information retrieval models to rank the web page based on similarity values. Term weighting schemes are the significant of an information retrieval system and it is query used in document ranking. Tf-idf ranked calculates the term weight according to users query on basis of term which is including in documents. When user enter query it will find the documents in which the query terms are included and it will count the term calculate the Tf-idf according to the highest weight of value it will gives the ranked documents.

\section{KEYWORD}

Information retrieval, term frequency - inverse frequency, vector space model, Cosine similarity

\section{INTRODUCTION}

In the information retrieval (IR) system documents are ranked optimally by using user's query to find out the relevant documents from large data base or form dataset [21].When the user gives a query, the index is consulted to archives the most relevant documents. The relevant documents are then ranked significance of their degree of relevance. Majority of internet users rely on search engines for extracting information by providing a query from any large dataset. These queries are processed by the search engines and a certain information retrieval or mining algorithm is applied to obtain the cluster of documents related to the query. After the retrieval of these documents, an important task is to present these documents in a list where documents at the top are the ones considered more relevant for the user. This task is called ranking of documents [15].Information retrieval system is a set of documents to discover convenient information equivalent to a user's query. In information retrieval basically data can be fetching from web structure information that can be type of content, pictures, graph etc. Several components make this task challenging :(i) normally unstructured information is in document database; (ii) reports are typically composed in unconstrained characteristic dialect; iii) regularly, the documents cover extensive variety range of subjects. There are various information model used .one of them is vector space model. It is a model for representing text documents or any other items as vectors of identifiers [17]. It is utilized as a part of information filtering, information retrieval, indexing and relevancy rankings. relevance rankings of documents in a keyword search can be calculated, using the suppositions of document equivalence theory, by comparing the deviation of angles between each document vector and the main query vector where the query is represented as the equivalent of vector as the documents. The vector space model technique can be partitioned into three stages. The main stage is the document indexing where content relevance terms are extracted from the document text. The second stage is the weighting of the indexed terms to enhance retrieval of documents relevant to the user. In the last stage, rank of the documents archives as per the query comparability value [4] [7]. Documents and queries are shows as vector

$$
\begin{aligned}
& d_{j}=\left(w_{1, j}, w_{2, j}, \ldots \ldots w_{t, j}\right) \\
& q=\left(w_{1, q}, w_{2, q}, \ldots \ldots w_{n, q}\right)
\end{aligned}
$$

There are dissimilar techniques for computing these values, which are known as (term) weights, have been produced. One of the best known schemes is Tf-idf weighting. A main advantage is that it is simple model based on linear algebra, ranking documents according to their possible relevance. Their term-weighting schemes enhance retrieval performance. Its partial matching procedure permits retrieval of documents that approximate the query conditions. 


\section{TF-IDF VECTOR SPACE MODEL}

In Information retrieval Tf-Idf is known as term frequency and inverse document frequency. It is a common method to assess how a word is required a document. It is commonly used as weighting factor in information retrieval. Tf-Idf is also a very interesting method to convert the textual representation of information into a Vector Space Model (VSM). The weight of term in document vector can be determined using method [14]. The weight of term is measured sometimes term $\mathrm{j}$ obtain in the document $\mathrm{i}$ (the term frequency) and tdf (the inverse document frequency) [7]. The weight of a term $j$ in the document $i$ is given by

$$
\begin{aligned}
\qquad w_{i, j} & =\text { freq }_{i, j} \times i d f \\
\text { And } \quad \text { idf } i & =\log \frac{N}{n_{i}}
\end{aligned}
$$

\section{MOTIVATION}

Research in document ranking is motivated not only by the challenges that are system faces but there are various reasons are listed below which motivated to do research in this area. The nature of most data on the Web is so unstructured that they can only be understood by humans, but the amount of data is so huge that they can only be processed efficiently by machines. From that large amount of data its difficult to find the relevance document according to user requirement. Different model used for information retrieval among of that vector space model is used for the information retrieval, indexing and ranking. TfIdf, term frequency-inverse document frequency ranker is a popular mechanism to calculate the relevance of very large documents. In traditional TfIdf ranker would calculate the weight of each document with respect to the words from the given query. This technique used when the words from the document is shared. But, due to the richness of natural language, a query can be expressed in different ways by different users. This mechanism is in which it retrieves the concepts which are most similar to the query from user.

\section{PROPOSED SYSTEM}

The proposed system is about ranking of different type of documents. Ranking is the process of ordering a set of items in order to show the most relevant first. In Ranking is the core of an Information Retrieval system because we need to know in what order to present the returned documents to the user. We are going to develop document ranking method for research data. Here use Vector space model for ranking the document. generally ranking method doesn't include phrase word at time of for selection. In our proposed method we will decide phrase word and also synonyms as term and we calculate TF-IDF to generate vector matrix. Tf-idf ranked calculates the term weight according to users query on basis of term which are include in documents. When user enter query it will find the documents in which the query terms are included and it will count the term calculate the TF-IDF according to the highest weight of value it will gives the document ranked.

\section{ALGORITHM STEPS: \\ Input : User Query \\ Output : Ranked Documents}

\section{Parameters :}

QTL : Query term list

Rc : Reading Counter

TF : Term Frequency

IDF : Inverse Document Frequency

Step 1 : Read the Query/Read the Document Decide format of Documents (Pdf, Docx, Txt etc.)

Step 2 : Read the documents Text and Remove Stop Words (is, are, that etc.)

(SMARTWORD List API)

Step 3 : Inialize QueryTermList (QTL/DTL

(Document term list), Phrase Wordlist $=$ null, matrix[N][QTsize] to 0 ,

Reading Counter to 0

Step 4 : Spilt and add each term to QTL

Find the Phrase Word in Query(E.g. HOT DOG)

Add Phrase words to Phrase Wordlist

Step 5: Remove the Single Words used in Phrase word from QTL

Append Phrase Word List to QTL

Find the synonyms of each term using word net API (Eg.BIG=HUGE) 
International Journal of Trend in Scientific Research and Development, Volume 1(4), ISSN: 2456-6470 www.ijtsrd.com

Step 6 : sIf synonyms not in list then add to list

Get the Size of Document List N (Metrics

Rows) ( $\mathrm{N}$ no of rows and $\mathrm{Tf}$ is Colum $)[\mathrm{m}][\mathrm{n}]$

While Rc $<\mathrm{N}$

null

$\&$ set Document Term List to
Step 8 : Calculate TF and IDF $[\mathrm{m}][\mathrm{n}]$

Step 9: Depend on the maximum value Rank the Document

Step 10 : Update Index

Step 7 : For each Term in Document Term List

Update the Matrices based on TF

End for

end while

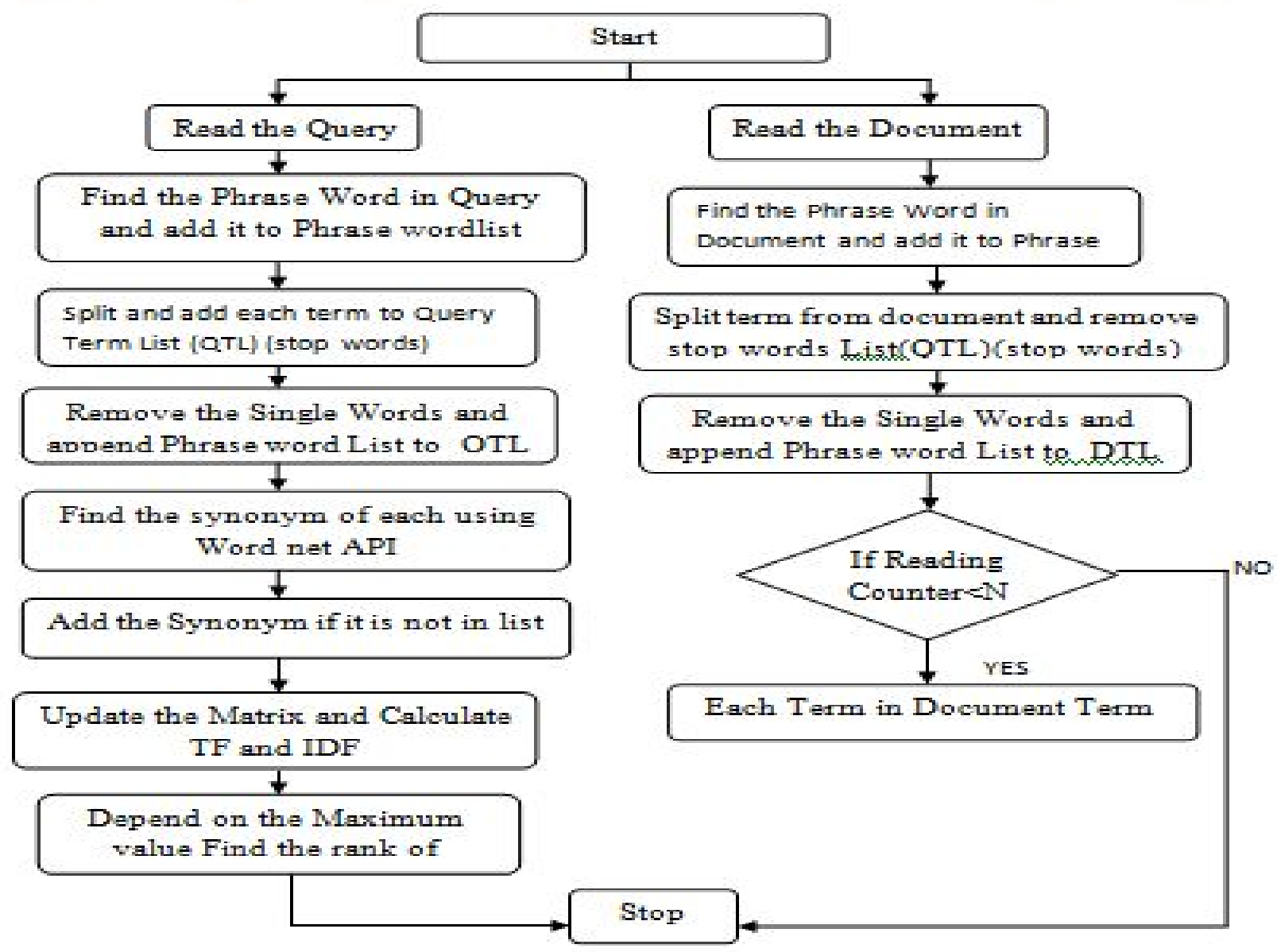

Fig. Flow of proposed work

\section{EVALUATION MEASURES}

Document ranking can be valued on various measures few of them are discussed in this section.
1) Precision - Precision measures how many of the items that the system identified were actually correct, regardless of whether it also failed to retrieve correct items. [24]

Precision $=\mathbf{t p} /(\mathbf{t p}+\mathbf{f p})$ 
where,

tp $=$ True Positive, case was positive and predicted positive

$\mathrm{fp}=$ False Positive, case was negative but predicted positive

2) Recall - Recall measures how many of the items that should have been identified actually were identified, regardless of how many spurious identifications were made. [24]

Recall $=\mathbf{t p} /(\mathbf{t p}+\mathbf{f n})$

where,

tp $=$ True Positive, case was positive and predicted positive

$\mathrm{fn}=$ False Negative, case was positive but predicted negative

3) F-Measure - The F-measure is often used in conjunction with Precision and Recall, as a weighted average of the two.

F-Measure $=2 *(\mathbf{P} * \mathbf{R}) /(\mathbf{P}+\mathbf{R})$

where,

$\mathrm{P}=$ Precision

$\mathrm{R}=$ Recall

4) Accuracy - Accuracy is the proportion of true results (both true positives and true negatives) among the total number of cases examined.

Accuracy $=(\mathbf{t p}+\mathbf{t n}) /(\mathbf{t p}+\mathbf{t n}+\mathbf{f n}+\mathbf{f p})$

where,

tp $=$ True Positive, case was positive and predicted positive

tn $=$ True Negative, case was negative and predicted negative

fn $=$ False Negative, case was positive but predicted negative

$\mathrm{fp}=$ False Positive, case was negative but predicted positive

\section{EXPERIMENTAL RESULTS}

To measure the performance of the proposed system the parameters available are discussed in the above section. It has been compared with the Time Complexity of ranked document. That is shown in Figure 5.1

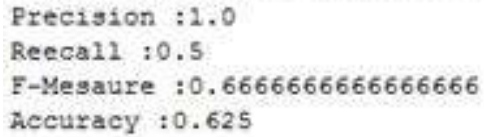

Fig. Documents Rank Time

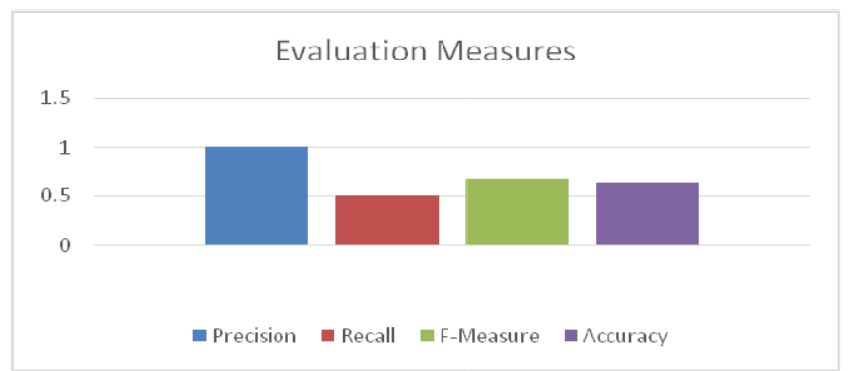

Fig. Evaluation Measures Result

Above figure shows the evaluation measures Precision, Recall ,F-measure and accuracy. Accuracy minimum time is 0.6 and in that time query will be exacting and gives the ranked documents.

\section{LITERATURE SURVEY}

In 2013 the researcher Jiaul H. Paik is represents a novel TF-IDF term weighting scheme. The suggest term weighting scheme has two feature of within document term frequency assign to discover the importance of a term. Experiments done at the huge amount of TREC news and web collection data and proposed that the out performs five state of the art retrieval model is significance and consistent. And its shows that proposed model better than the existing models [6].

T.Suganya and M.Ravichandran proposed a method in e-learning rank ordering the documents according to their individual term relevance degree using possibility approach and vector-based technique method. This proposed system provides highly relevant learning materials to the learner and it recommends the items based on individual term 
relevance with respect to the query specified by the user [5].

In proposed vector space model used in XML document ranking suggest by Weimin He and Teng Lv. They proposed effectively rank Xml document and also differentiate the framework with Lucene to demonstrate their extended TF*IDF is successful and it is effective ranking than existing XML search engine Lucene [15].

Premalatha.R and Srinivasan.S emphasis, on Information retrieval for Tamil literary document using the model vector space. they approaches in text processing in information retrieval. In their system explore that can be divided into three categories, Main topic search, Subtitle search and Keyword search. So the system would explore necessitate information rapidly mainly using the vector space model, that illustrate documents as vectors. It would be applicable for all Tamil literates and understudies to look and learn [2]

In their research Vaibhav Kant Singh and Vinay Kumar Singh is proposed a vector space model using in information retrieval. In that individual document and user query is represented as a vector based against the vocabulary and Calculating similarity measure and than Ranking the documents for relevance and other variant of VSM that Term weighting, Normalized term frequency(tf) and Inverse document frequency (idf) is shows in their system[1].

Bo $\mathrm{Yu}$ and Guoray Cai is recommend a dynamic document ranking scheme join thematic and geographic pertinence measures on a for each query premise. They have been using Dempster-Shafer's theory to gather the two different sources of ranking verification and evaluate the different web document data set. Which can be either news stories or blog and it can be fetch from the web data [19].

Dik Lun Lee, Huei Chuang and Kent E.Seamons is proposed that Using various interpretation of the vector-space model for text retrieval queries, they optimal balance between processing efficiency and retrieval effectiveness as expressed in relevant document rankings. They using six different vector method and their retrieval effectiveness [20].

\section{CONCLUSION}

In this paper we conclude that on the basis of query document ranking is utilized for search relevant document so that information retrieval is a process of searching and retrieving the knowledge based information from collection of documents. for that their distinctive model is used with its advantages. documents are comparing with the input query. Vector space model is used in information filtering, information retrieval and relevancy ranking of documents. ranked first. Tf-idf ranked calculate the term weight according to users query on basis of term which are include in documents. When user enter query it will find the documents in which the query terms are included and it will count the term calculate the TF-IDF according to the highest weight of value it will gives the document ranked

Future research include variants of Tf-Idf and weighting of query word can be find and in future using special character also used in query searching..

\section{REFERENCES}

[1] Singh, Vaibhav Kant, and Vinay Kumar Singh. "VECTOR SPACE MODEL: AN INFORMATION RETRIEVAL SYSTEM." Int. J. Adv. Engg. Res. Studies/IV/II/Jan.-March 141 (2015): 143.

[2] Premalatha, R., and S. Srinivasan. "Text processing in information retrieval system using vector space model." Information Communication and Embedded Systems (ICICES), 2014 International Conference on. IEEE, 2014

[3] Khan, Junaid. "Comparative study of information retrieval models used in search engine." Advances in Engineering and Technology Research (ICAETR), 2014 International Conference on. IEEE, 2014.

[4] Singh, Jitendra Nath, and Sanjay K. Dwivedi. "Comparative Analysis of IDF Methods to Determine Word Relevance in Web Document." International Journal of Computer Science Issues (IJCSI) vol 11 (2014): 59-65.

[5] T. Suganya and M. Ravichandran, "Ranking Documents in IR Using Vector Based Ordering In E-Learning," vol. 3, no. 3, 2014.

[6] Paik, Jiaul H. "A novel TF-IDF weighting scheme for effective ranking."Proceedings of the 36th international ACM SIGIR conference on Research and development in information retrieval. ACM, 
2013.

[7] J. N. Singh, "A Comparative Study on Approaches of Vector Space Model in Information Retrieval," pp. 37-40, 2013

[8] Asadi, Nima, and Jimmy Lin. "Document vector representations for feature extraction in multistage document ranking." Information retrieval 16.6 Springer (2013): 747-768.

[9] Bhatia, Parul Kalra, Tanya Mathur, and Tanaya Gupta. "Survey Paper on Information Retrieval Algorithms and Personalized Information Retrieval Concept." International Journal of Computer Applications 66.6 (2013).

[10] Sharma, Manish, and Rahul Patel. "A Survey on Information Retrieval Models, Techniques And Applications." International Journal of Emerging Technology and Advanced Engineering, ISSN (2013): 2250-2459.

[11] Sharma, Aditi, Nishtha Adhao, and Anju Mishra. "A survey: Static and dynamic ranking." International Journal of Computer Applications 70.14 (2013): 7-12.

[12] Wang, Shuaiqiang, et al. "Adapting vector space model to ranking-based collaborative filtering." Proceedings of the 21st ACM international conference on Information and knowledge management. ACM, 2012.

[13] Raman, Shivangi, Vijay Kumar Chaurasiya, and Swaminathan Venkatesan. "Performance comparison of various information retrieval models used in search engines." Communication, Information \& Computing Technology (ICCICT), 2012 International Conference on. IEEE, 2012.

[14] Zhang, GuanHong. "Sentence alignment for web page text based on vector space model." Computer Science and Information Processing (CSIP), 2012 International Conference on. IEEE, 2012.

[15] He, Weimin, and Teng Lv. "Extending vector space model for XML ranking."Applications of Digital Information and Web Technologies (ICADIWT), 2011 Fourth International Conference on the. IEEE, 2011.

[16] Barrera, Araly, and Rakesh Verma. "A ranking-based approach for multiple-document information extraction." University of Houston (2010).

[17] Khankasikam, Krisda. "A comparison of information retrieval models applied to Thai digital library." Computer and Automation Engineering (ICCAE), 2010 The 2nd International
Conference on. Vol. 1. IEEE, 2010.

[18] Xu, Huaiyu, et al. "An intelligent project agency for web-3D virtual trading community based on google earth." Computer Science and Information Technology, 2009. ICCSIT 2009. 2nd IEEE International Conference on. IEEE, 2009.

[19] $\mathrm{Yu}, \mathrm{Bo}$, and Guoray Cai. "A query-aware document ranking method for geographic information retrieval." Proceedings of the 4th ACM workshop on Geographical information retrieval. ACM, 2007.

[20] Dik L. Lee, Huei Chuang, Kent Seamons, "Document Ranking and the Vector-Space Model", IEEE Software vol.14, no. 2, pp. 67-75, March/April 1997, doi:10.1109/52.582976

[21] Salton, Gerard, Anita Wong, and ChungShu Yang. "A vector space model for automatic indexing." Communications of the ACM 18.11 (1975): 613-620.

[22] R. Azhar-ul-haq, "A Review :Ranking documents using Ranking Algorithms \& Techniques." 